\title{
A Farmland Monitoring System Based on Web and Wireless Sensor Networks(WSNs)
}

\author{
Kaicheng Zhen ${ }^{1, ~ a ~ a n d ~ L i n l i ~ Z h o u ~}{ }^{1, b, *}$ \\ ${ }^{1}$ Institute of Intelligent Machines, Chinese Academy of Sciences, Hefei 230031, China. \\ a kczhen@mail.ustc.edu.cn, binlizhou@iim.ac.cn
}

Keywords: Wireless sensor networks, farmland monitoring, Web applications.

\begin{abstract}
In this paper, wireless sensor network architecture for farmland is presented in order to achieve lower management costs from the aspect of environmental parameters monitoring. According to the analysis of the features of farmland environment, a practical and low-cost farmland monitoring system is designed based on wireless sensor network technology in order to monitor key environmental parameters such as the temperature, humidity and wind speed. A Web application is implemented that allows a user to view the data which are stored in database server through browsers.
\end{abstract}

\section{Introduction}

Wireless sensor networks(WSNs) have successfully been used in many fields. This fields include forest-fires surveillance[1], building environmental monitoring [2], health care[3], smart cities[4,5], and some other fields[6,7]. Recent advances in wireless communications and electronics have enabled the development and production of low-cost, low-power, and multi-functional sensors that are small in size and communicate in short distances. This development of WSNs make it easier to implement a larger-scale monitor system.

In the context of the growing demand for food for mankind, increasing food production and reducing production costs is an inevitable trend. Using WSNs in the agricultural production process can achieve this demand. Therefore, agriculture is an important application of WSNs. Some literature call such this agriculture monitoring system as precision agriculture[8,9]. And reference[10] details the application of WSNs in agriculture, such as irrigation[11], fertilization, pest control, horticulture.

Moreover, according to [12,13], the Internet is becoming the basic carrier for interconnecting devices into information system and therefore, integrating WSNs in these monitoring systems over the Internet is a fundamental issue. A web application can easily show acquisition data to users with easy-to-understand graphical user interface(GUI). Furthermore, with the development of mobile Internet, users visit the website through mobile phone, pad, and other handheld devices conveniently. Paper [14] introduced a method for integrating WSNs with web. Jang et al., design web application to This work aims to develop wireless sensor node which can measure farmland environment parameters, implement a communication network that can handle data acquisition, transmission and storage, and design a web application that demonstrate this data to stakeholders(farmers, farmland managers, etc.). Sensor node consisting of temperature, humidity, air press, wind speed sensors to monitor the environmental parameters which would affect the crop production. Database server and web server is needed, so that user can visit the illustration of the parameters.

\section{Data acquisition}

Several agriculture parameters such as temperature and humidity of the air and soil, air press, affect the crop growth. WSNs provide monitoring and acquisition of various measured parameters. Thus, to implement a farmland monitoring system, sensor node data must be processed, packaged and transmitted by WSNs. 
A typical ZigBee-based wireless sensor network architecture consists of a sensor node, a sink node(gateway), and a data storage devices. As shown in Fig. 1., a large number of sensor nodes randomly arranged in the vicinity of the monitoring area, through the Ad-Hoc network to complete the perception and data sampling function. The communication between the sensor nodes follows the ZigBee communication protocol. The monitoring data is transmitted hop by hop along the other nodes. The monitoring data may be routed through multiple nodes and then routed to the gateway after multipath. Finally, the monitoring data obtained by the gateway arrives at the database server through the TCP/IP protocol. After the data is saved in the database server, user can process and visualize the data , configure and manage the sensor network, publish the monitoring task and collect the monitoring data through the gateway.The gateway can be configured to have the storage function, its built-in SQLite database can store 500 thousand sensor data, thus avoiding the data loss caused by network interruption.

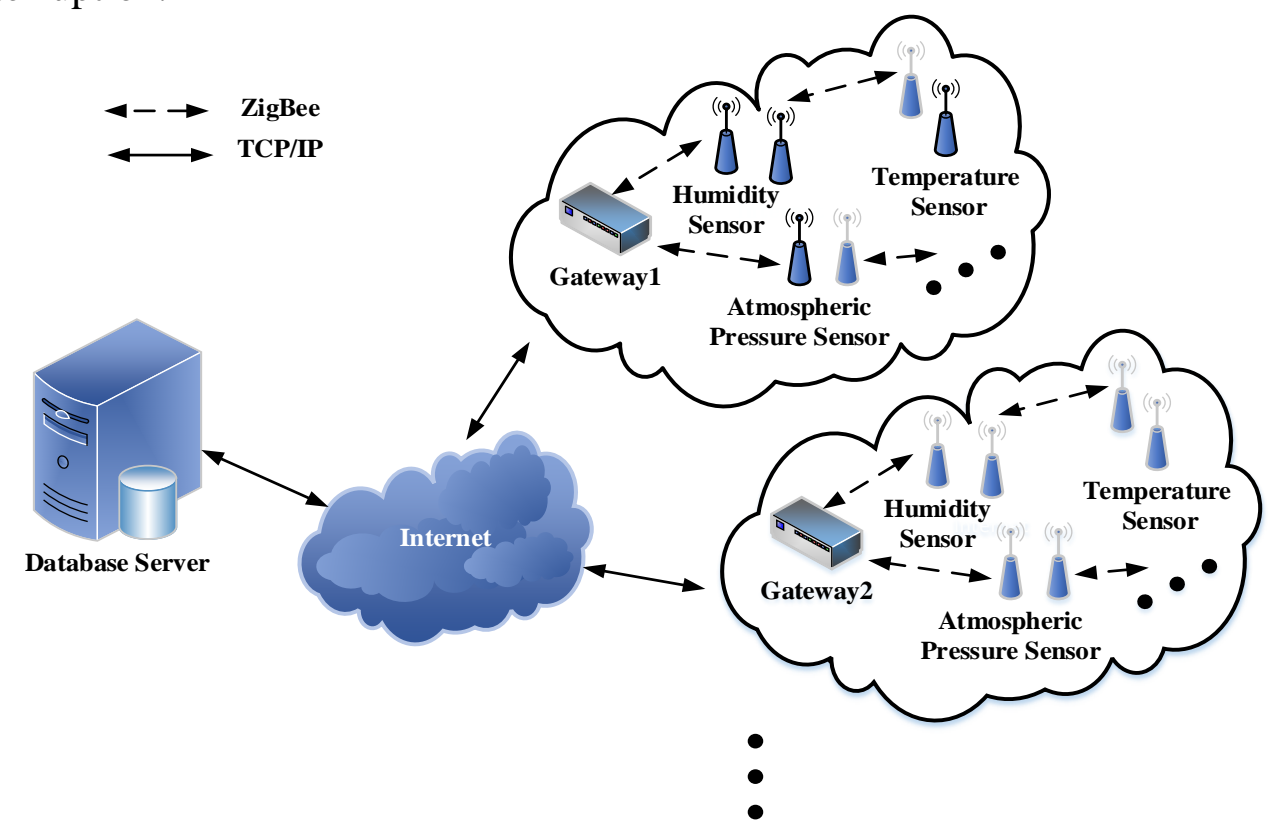

Fig. 1 The diagram of the WSNs monitoring system deployed in the farmland

In an open environment, the communication distance of gateway is about $800 \mathrm{~m}$.In addition, one gateway can only work with 64 sensors. The parameters of the gateway are shown in Table 1 . Taking into account the acreage of the farmland, the communication distance of gateway, communication bandwidth and communication interference, the farmland is divided into zones and each of zones forming a sub-network.

Each gateway sends a record to the database server every minute and each record contains the latest data of all sensor nodes in the sub-network, the equipment ID of the gateway and the send time of the record. Every sub-network contain two temperature sensor to monitor air and soil temperature, two humidity sensor to monitor air and soil humidity, a air press sensor and a wind speed sensor.

Table 1 Gateway technology parameters.

\begin{tabular}{cc}
\hline Name & Description \\
\hline Supply voltage & DC $20 \sim 30 \mathrm{~V}$ \\
Average power consumption & $1.7 \mathrm{~W}$ \\
Communication mode & Ethernet/WIFI/3G/4G \\
Working model & Server/Client \\
Protocol type & Modbus TCP \\
ZigBee frequency point & 0X00 0X0F \\
ZigBee network ID & 0X0000 0XFFEE \\
ZigBee communication distance & $800 \mathrm{~m}($ In the open area) \\
\hline
\end{tabular}

The database server is an independent computer designed to store data uploaded by WSNs.The database was created using MySQL, a free database server software. The structure of the database was devised to help provide all of the necessary metadata to describe the data generated by the sensor 
network and to store the data for any suite of sensors placed on a node. The database comprises 7 tables. Date generated by WSNs are stored in the DateRecrived table. Each entry contains a counter to provide a unique identification of the data value(Id), and a equipment number(equipNo) to identify which gateway are belong to.

\section{Data visualization}

Although the data is already stored on the database server, it is still difficult for the user to view it. In order to demonstrate this data to users, an application is need to be implemented. We aimed to develop a system by which the data were accessible from anywhere, therefore, developed to access the sensor database over the Internet. The scripting software to access the database and display results was written by JAVA and HTML.JavaScript charts was used in the visualization. It lets the user modify the database to add more sensor nodes and allows him to query the database to find necessary data. Fig. 2 show how user visit the data in the database server.
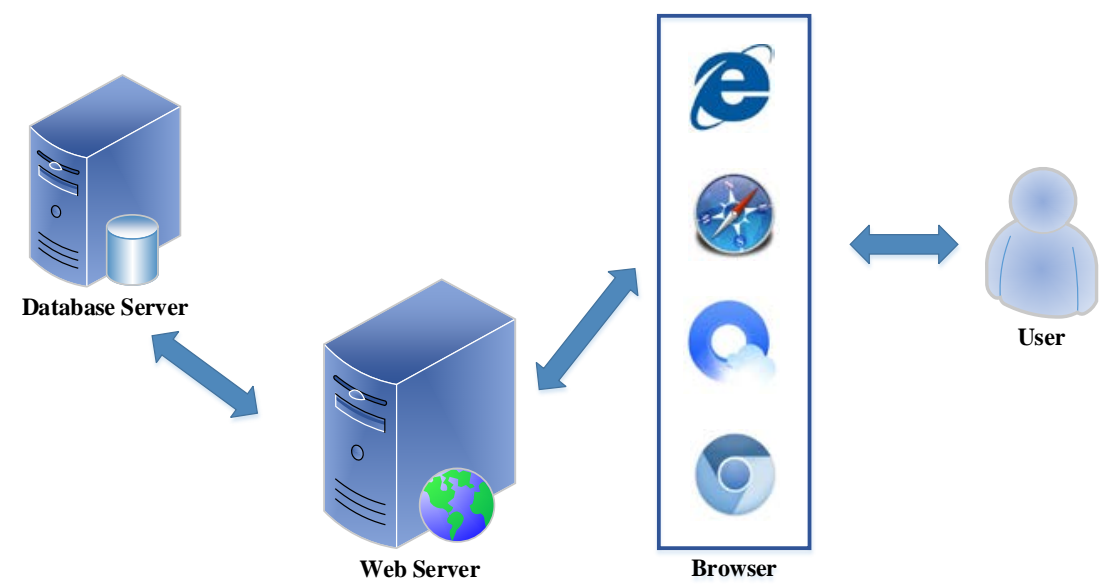

Fig. 2 User access the data through browser

The most important application of this web is to display the data uploaded by WSNs. Further more, there are some humanized function contained. The alarm system supports the user to set up the upper and lower limits of the various environmental parameters for each node. Once the sensor network monitors the value not in the range, it will trigger the alarm system. And then the alarm system will send text messages to the relevant personnel on the phone. The data statistics analysis function will complete the analysis and statistics of the existing data, such as count the daily maximum temperature and humidity of 10 nodes, compare with yesterday's real-time environmental parameters. The information center allows the relevant stakeholders to access and publish various production plans and other information.

\section{Summary}

This paper introduced a the implementation of a farmland monitoring system. WSNs is responsible for monitoring, acquisition and transmission of environmental parameters of farmland. The database server is responsible for data storage and the web server is executing web application and illustrating the data to the farmland stakeholders.The environmental parameters such as temperature and humidity should be continually monitored in order to adjust crop growth conditions. One such database design is provided here as a suggested method of structuring the data for monitoring system application. To access the data, this work relies on a web-based viewer that allows a user to view the data via a conventional web browser. This user interface requires no advanced knowledge of programming, so the sensor network data will be available to users with limited skills in programming. 


\section{Acknowledgment}

This work was supported by the National Science \& Technology Pillar Program during the 12th Five-year Plan Period (Grant No. 2015BAD18B05).

\section{References}

[1] Son B, Her Y S. A Design and Implementation of Forest-Fires Surveillance System based on Wireless Sensor Networks for South Korea Mountains[J]. International Journal of Computer Science and Network Security (IJCSNS, 2006, 6(9B):124-130.

[2] Jang W S, Healy W M, Skibniewski M J. Wireless sensor networks as part of a web-based building environmental monitoring system[J]. Automation in Construction, 2008, 17(6):729-736.

[3] Hakimi A, Hassan N, Anwar K, et al. Development of real-time patient health (jaundice) monitoring using wireless sensor network[C]// International Conference on Electronic Design. 2016:404-409.

[4] Samaras I, Gialelis J, Koubias S. Using web services-based wireless sensor networks for estimating the free size of parking places[C]// International Conference on Telecommunications. IEEE, 2016:1-5.

[5] Rashid B, Rehmani M H. Applications of wireless sensor networks for urban areas: A survey[J]. Journal of Network \& Computer Applications, 2016, 60:192-219.

[6] Judge M A, Manzoor A, Ahmed F, et al. Monitoring of Power Transmission Lines Through Wireless Sensor Networks in Smart Grid[C]// International Conference on Innovative Mobile and Internet Services in Ubiquitous Computing. Springer, Cham, 2017:162-170.

[7] Casey K, Lim A, Dozier G. A Sensor Network Architecture for Tsunami Detection and Response[J]. International Journal of Distributed Sensor Networks, 2008, 4(1):28-43.

[8] Srbinovska M, Gavrovski C, Dimcev V, et al. Environmental parameters monitoring in precision agriculture using wireless sensor networks[J]. Journal of Cleaner Production, 2015, 88:297-307.

[9] Flores K O, Butaslac I M, Gonzales J E M, et al. Precision agriculture monitoring system using wireless sensor network and Raspberry Pi local server[C]// Region 10 Conference. IEEE, 2017:3018-3021.

[10] Aqeel-Ur-Rehman, Abbasi A Z, Islam N, et al. A review of wireless sensors and networks' applications in agriculture[J]. Computer Standards \& Interfaces, 2014, 36(2):263-270.

[11]Deshpande V A, Prasad J P. Automated Irrigation System Using a Wireless Sensor Network and GPRS Module[C]// International Conference on Signal Processing, Image Processing and Vlsi. 2015.

[12] Samaras I K, Hassapis G D, Gialelis J V. A Modified DPWS Protocol Stack for 6LoWPAN-Based Wireless Sensor Networks[J]. IEEE Transactions on Industrial Informatics, 2013, 9(1):209-217.

[13]Torrisi N M. Monitoring Services for Industrial[J]. Industrial Electronics Magazine IEEE, 2011, 5(1):49-60.

[14]Colitti W, Steenhaut K, De N. Integrating Wireless Sensor Networks with the Web[J]. Proceedings of Workshop on Extending the Internet to Low Power \& Lossy Networks, 2011. 